\title{
USE OF REX CONTROL SYSTEM FOR THE BALL ON SPOOL MODEL
}

\author{
Štěpán Ožana - Martin Pieš - \\ Radovan Hájovský — Tomáš Dočekal *
}

\begin{abstract}
This paper deals with the design and implementation of linear quadratic controller (LQR) for modeling of Ball on Spool. The paper presents the entire process, starting from mathematical model through control design towards application of controller with the use of given hardware platform. Proposed solution by means of REX Control System provides a high level of user comfort regarding implementation of control loop, diagnostics and automatically generated visualization based on HTML5. It represents an ideal example of a complex nonlinear mechatronic system with a lot of possibilities to apply other types of controllers.
\end{abstract}

K e y w o r d s: educational model, control design, control system, LQR, REX

\section{INTRODUCTION}

Laboratory model of Ball on spool was created for enhancement of educational activities at the Department of Cybernetics and Biomedical engineering. The ball is placed on the spool which is connected to the shaft and drive. The main control task is to keep the ball at the top (unstable) upright position by appropriate control provided by the drive affecting the spool. The "swing-up" control task is beyond the scope of this paper, although it is currently in progress.

Physical setup of the model is presented in Fig. 1. It is composed of the drive and shaft connected to spool with a ball on the top, while the feedback is provided by ultrasonic distance sensor and IRC position sensor. Inside the assemble unit there is a single-board computer ALIX 2D13, B-R X-20 I/O modules, control unit Maxon DES $50 / 5$ and accessories.

Detail architecture of laboratory model of Ball on Spool is shown in Fig. 1. The model is controlled by SBC (Single Board Computer) ALIX, which communicates with remote IO modules of X20 series made by B\&R Automation. These modules process all of the input and output signals present in the physical system. Rotation of the spool is provided by servo motor whose control unit takes care of torque (current) regulation loop. It is preset so that its parameters needn't change. Required torque (set-point) is handed over to the control unit by analogue output of D/A converter (X20AO2622 module), and in reverse direction the control systems receives information on ball position measured by ultrasonic sensor (signal is brought to the A/D converter of X20AI4222 analogue input module) and it also receives pulses of incremental counter determining motor shaft position (brought to the
X20DC1176 digital counter module). There are two digital outputs (from X20DO4322 module), that is for enabling motor rotation, and for lightning the inner space of the model box. The control algorithm itself is running under REX Control System on ALIX single-board computer with operating system Linux. Precise execution of particular tasks and communication by Ethernet POWERLINK is provided by its central core (RexCore) with appropriate driver. Supervising layer could be standard industrial PC which serves for control, archiving, visualization, configuration and diagnostics.

\section{MATHEMATICAL BACKGROUND}

The presented model of Ball on Spool is a complex nonlinear model. Its mathematical description is based on physical analysis according Fig. 2 with parameters listed in Tab. 1. At first the full nonlinear model is introduced, then it is turned into a simplified nonlinear model (composed of 2 separate parts) and linearized. Once the linearized model is achieved in the form of state-space description, it is followed by design of LQR controller.

Parameters and variables of the model. Determination of mathematical model is based upon parameters listed in table Tab. 1 achieved by experimental measurements.

Full nonlinear model. Full nonlinear model of Ball on Spool is based on general Lagrange equations of the $2^{\text {nd }}$ type. Detail derivation is beyond the scope of this paper, it is introduced in [1]. The paper presents final form of the equations (1) and (2). For practical implementation of control algorithm regarding chosen hardware platform,

* VSB-Technical University of Ostrava, Department of Cybernetics and Biomedical Engineering, stepan.ozana@vsb.cz, martin.pies@vsb.cz, radovan.hajovsky@vsb.cz, jiri.haska@vsb.cz 


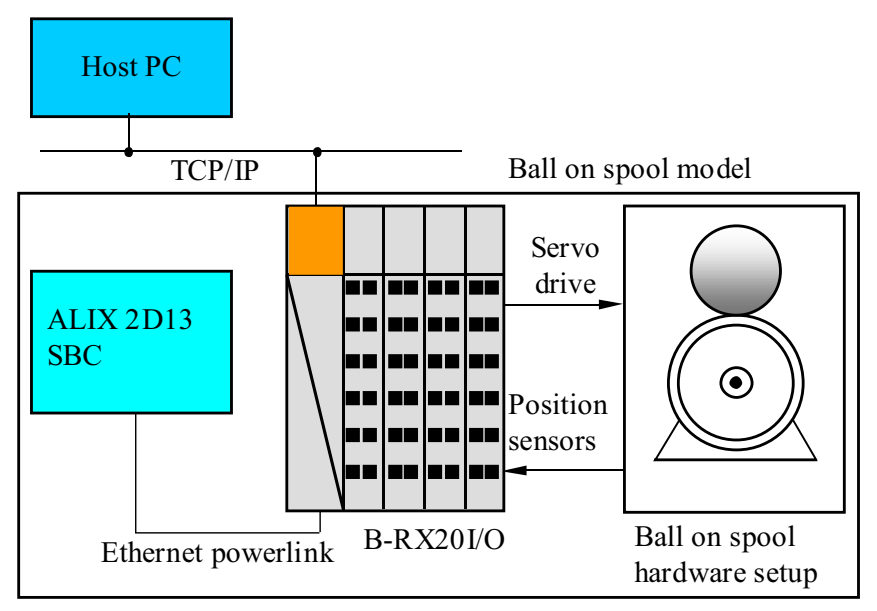

Fig. 1. Physical model of Ball on spool with interconnection of hardware parts

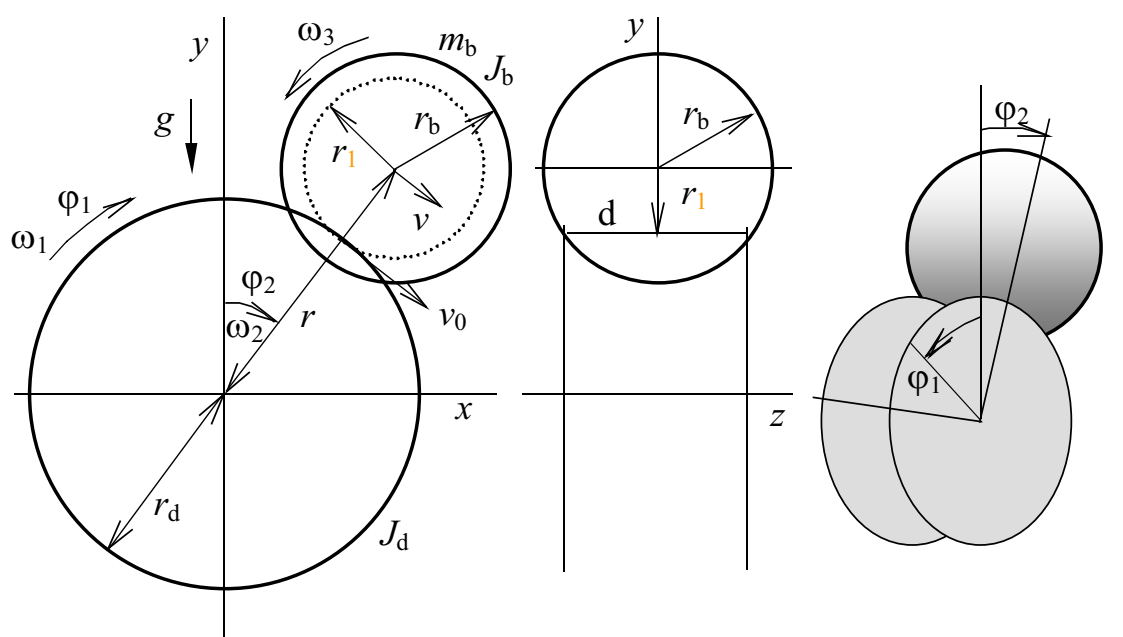

Fig. 2. Situation scheme with parameters

the paper presents a simplified model introduced in the following sections.

$$
\ddot{\varphi}_{1}\left(J_{d}+J_{b} \frac{r_{d}^{2}}{r_{r}^{2}}\right)-\ddot{\varphi}_{2}\left(J_{b} \frac{r_{d}^{2}}{r_{r}^{2}}\right)=Q
$$

$-\ddot{\varphi}_{1}\left(J_{b} \frac{r_{d}^{2}}{r_{r}^{2}}\right)+\ddot{\varphi}_{2}\left(m_{b} r^{2}+J_{b} \frac{r_{d}^{2}}{r_{r}^{2}}\right)-M_{b} g r \sin \varphi_{2}=-Q_{f}$.

Solution for $\ddot{\varphi}_{1}$ and $\ddot{\varphi}_{2}$ leads to a set of equations

$\ddot{\varphi}_{1}=\frac{m_{b} r_{r}^{2} r^{2} Q+m_{b} J_{b} r_{d}^{2} g r \sin \varphi_{2}+J_{b} r_{d}^{2} Q-J_{b} r_{d}^{2} Q_{f}}{m_{b} J_{d} r_{r}^{2} r^{2}+m_{b} J_{b} r_{d}^{2} r^{2}+J_{b} J_{d} r_{d}^{2}}$ $\ddot{\varphi}_{2}=\frac{1}{m_{b} J_{d} r_{r}^{2} r^{2}+m_{b} J_{b} r_{d}^{2} r^{2}+J_{b} J_{d} r_{d}^{2}}\left(m_{b} g J_{d} r_{r}^{2} r \sin \varphi_{2}\right.$

$$
\left.+m_{b} g J_{b} r_{d}^{2} r \sin \varphi_{2}+J_{b} r_{d}^{2} Q-J_{b} r_{d}^{2} Q-J_{d} r_{r}^{2} Q_{f}\right) \text {. }
$$

Having introduced variables $x_{1}=\varphi_{1}, x_{2}=\dot{\varphi}_{1}=$ $\dot{x}_{1}, x_{3}=\varphi_{2}, x_{4}=\dot{\varphi}_{2}=\dot{x}_{3}$, the following state-space description is achieved

$$
\begin{aligned}
& \dot{x}_{1}=x_{2}, \\
& \dot{x}_{2}=\frac{m_{b} r_{r}^{2} Q+m_{b} J_{b} r_{d}^{2} g r \sin x_{3}+J_{b} r_{d}^{2} Q-J_{b} r_{2}^{2} Q_{f}}{m_{b} J_{d} r_{r}^{2} r^{2}+m_{b} J_{b} r_{d}^{2} r^{2}+J_{b} J_{d} r_{d}^{2}}, \\
& \dot{x}_{3}=x_{4},
\end{aligned}
$$

$$
\begin{aligned}
\dot{x}_{4} & =\frac{1}{m_{b} J_{d} r_{r}^{2} r^{2}+m_{b} J_{b} r_{d}^{2} r^{2}+J_{b} J_{d} r_{d}^{2}}\left(m_{b} g J_{d} r_{r}^{2} r \sin x_{3}\right. \\
& \left.+m_{b} g J_{b} r_{d}^{2} r \sin x_{3}+J_{b} r_{d}^{2} Q-J_{b} r_{d}^{2} Q_{f}-J_{d} r_{r}^{2} Q_{f}\right) .
\end{aligned}
$$

This high-fidelity model represents dynamic behavior of mechatronic system very well, including friction between ball and spool represented by $Q_{f}=K_{f} \dot{\varphi}_{2}$. However, this model uses unknown drive moment $Q$, which would have to be determined by modeling entire dynamic 
Table 1. Parameters and variables of the model according [1]

\begin{tabular}{lll}
\hline Notation & Description & Unit/Value \\
\hline$\varphi_{1}, \omega_{1}$ & Angle and angular speed of spool disks & $\mathrm{rad} / \mathrm{s}, \mathrm{rad}$ \\
$\varphi_{2}, \omega_{2}$ & Angle and angular speed of rolling ball movement on the spool $\mathrm{rad} / \mathrm{s}, \mathrm{rad}$ \\
$r_{d}$ & Disk radius (spool face) & $0.09 \mathrm{~m}$ \\
$r_{b}$ & Ball radius & $0.085 \mathrm{~m}$ \\
$r_{b i}$ & Inner ball radius & $0.084 \mathrm{~m}$ \\
$r$ & Moment of inertia (discs) $J_{d}=\frac{1}{2} m_{s} r_{s}^{2}+m_{d} r_{d}^{2}+m_{c} r_{c}^{2}$ & $0.157 \mathrm{~m}$ \\
$J_{d}$ & Moment of inertia (ball) $J_{b}=\frac{2}{5} m_{b} \frac{r_{b}^{5}-\left(r_{b}-t\right)^{5}}{r_{b}^{3}-\left(r_{b}-t\right)^{3}} \approx \frac{2}{3} m_{b} r_{b}^{2}$ & $\mathrm{~kg} \mathrm{~m}^{2}$ \\
$J_{b}$ & Ball mass & $0.405 \mathrm{~kg}^{2}$ \\
$m_{b}$ & Time constant for velocity and torque loop & $0.015 \mathrm{~s}$ \\
$\tau$ & Friction coefficient & 0.3 \\
$K_{f}$ & Gravity constant & $9.81 \mathrm{~m} \mathrm{~s}^{-1} \mathrm{~kg}^{-2}$ \\
$g$ & Torque due to rolling ball friction & $\mathrm{N} \mathrm{m}$ \\
$Q_{f}$ & General drive torque & $\mathrm{N} \mathrm{m}$ \\
$Q$ & & \\
\hline
\end{tabular}

of the drive including torque control loop. Therefore, the model is adjusted and turned into simplified model, composed of two sub-systems: ball moving on spool, and velocity and torque control loop for drive and shaft connected to spool.

Simplified model - ball moving on spool ( $1^{\text {st }}$ part). Let's go back to (1) and (2). The first one determines the relation between angular acceleration and driving torque of the motor. Angular acceleration of the entire spool $\ddot{\varphi}_{1}$ is weighted sum of driving motor torque and angular acceleration of the ball on spool. During regulation of the motor, its behavior is fundamentally determined by a torque controller of the motor. Therefore angular acceleration $\ddot{\varphi}_{1}$ can be interpreted as an input of the simplified model and dynamics of the rest of the model can be derived from (2), using $Q_{f}=K_{f} \dot{\varphi}_{2}$, in the form of (5), or after linearization, by use of $\sin \varphi_{2} \approx \varphi_{2}$ for $\varphi_{2} \approx 0$ in matrix form as described by (6).

$$
\ddot{\varphi}_{2}=\frac{\ddot{\varphi}_{1} J_{b}\left(\frac{r_{d}^{2}}{r_{r}^{2}}\right)-K_{f} \dot{\varphi}_{2}+m_{b} g r \sin \varphi_{2}}{m_{b} r^{2}+J_{b} \frac{r_{d}^{2}}{r_{r}^{2}}},
$$

$$
\begin{array}{r}
{\left[\begin{array}{c}
\dot{x}_{1} \\
\dot{x}_{2} \\
\dot{x}_{3} \\
\dot{x}_{4}
\end{array}\right]=\left[\begin{array}{cccc}
0 & 1 & 0 & 0 \\
0 & 0 & 0 & 0 \\
0 & 0 & 0 & 1 \\
0 & 0 & \frac{m_{b} g r}{m_{b} r^{2}+J_{b} r_{d}^{2} / r_{r}^{2}} & -K_{f}
\end{array}\right]\left[\begin{array}{c}
x_{1} \\
x_{2} \\
x_{3} \\
x_{4}
\end{array}\right]} \\
+\left[\begin{array}{c}
0 \\
1 \\
0 \\
\frac{J_{b} r_{d}^{2} / r_{r}^{2}}{m_{b} r^{2}+J_{b} r_{d}^{2} / r_{r}^{2}}
\end{array}\right] \ddot{\varphi}_{1}
\end{array}
$$

Simplified model - velocity and torque control loop for drive and shaft with spool ( $2^{\text {nd }}$ part). This part can be identified by the first-order system $F(s)=\frac{1}{\tau s+1}$ where the transfer function is related to angular speed, between set-point value $\omega_{1 s p}$ and its output value $\omega_{1}$, see Fig. 3 . Determination of exact value of parameter $\tau$ is carried out by experimental measurements and it is beyond the scope of this paper.

Simplified model - composition. Having integrated $1^{\text {st }}$ and $2^{\text {nd }}$ parts of the simplified model, the following description is achieved (7), or (8) after linearization, by use of $\sin x_{3} \approx x_{3}$ for $x_{3} \approx 0$.

$$
\begin{aligned}
\dot{x}_{1} & =x_{2}, \\
\dot{x}_{2} & =-\frac{1}{\tau}+\frac{1}{\tau} \omega_{1 s p}, \\
\dot{x}_{3} & =x_{4} \\
\dot{x}_{4} & =-\frac{J_{b} r_{d}^{2} / r_{r}^{2}}{\tau\left(m_{b} r^{2}+J_{b} r_{d}^{2} / r_{r}^{2}\right)} x_{2}+\frac{m_{b} g r}{m_{b} r^{2}+J_{b} r_{d}^{2} / r_{r}^{2}} \sin x_{3} \\
& -K_{f} x_{4}+\frac{J_{b} r_{d}^{2} / r_{r}^{2}}{\tau\left(m_{b} r^{2}+J_{b} r_{d}^{2} / r_{r}^{2}\right)} \omega_{1 s p},
\end{aligned}
$$

$$
\begin{gathered}
{\left[\begin{array}{c}
\dot{x}_{1} \\
\dot{x}_{2} \\
\dot{x}_{3} \\
\dot{x}_{4}
\end{array}\right]=\left[\begin{array}{cccc}
0 & 1 & 0 & 0 \\
0 & -1 / \tau & 0 & 0 \\
0 & 0 & 0 & 1 \\
0 \frac{J_{b} r_{d}^{2} / r_{r}^{2}}{\tau\left(m_{b} r^{2}+J_{b} r_{d}^{2} / r_{r}^{2}\right)} & \frac{m_{b} g r}{m_{b} r^{2}+J_{b} r_{d}^{2} / r_{r}^{2}} & -K_{f}
\end{array}\right]\left[\begin{array}{l}
x_{1} \\
x_{2} \\
x_{3} \\
x_{4}
\end{array}\right]} \\
+\left[\begin{array}{c}
0 \\
1 / \tau \\
0 \\
\frac{J_{b} r_{d}^{2} / r_{r}^{2}}{\tau\left(m_{b} r^{2}+J_{b} r_{d}^{2} / r_{r}^{2}\right)}
\end{array}\right] \omega_{1 s p} \cdot(8)
\end{gathered}
$$

Having substituted values of all the parameters, the state-space description of simplified Ball on Spool model is according (9). This represents matrices A,B used for the LQR design.

$$
\begin{array}{r}
{\left[\begin{array}{c}
\dot{x}_{1} \\
\dot{x}_{2} \\
\dot{x}_{3} \\
\dot{x}_{4}
\end{array}\right]=\left[\begin{array}{cccc}
0 & 1 & 0 & 0 \\
0 & -66-.66671 & 0 & 0 \\
0 & 0 & 0 & 1 \\
0 & -17.2285 & 46.3365 & -0.3
\end{array}\right]\left[\begin{array}{l}
x_{1} \\
x_{2} \\
x_{3} \\
x_{4}
\end{array}\right]} \\
+\left[\begin{array}{c}
0 \\
66.6667 \\
0 \\
17.2285
\end{array}\right] \omega_{1 s p} .
\end{array}
$$


Velocity and torque control loop for drive and shaft

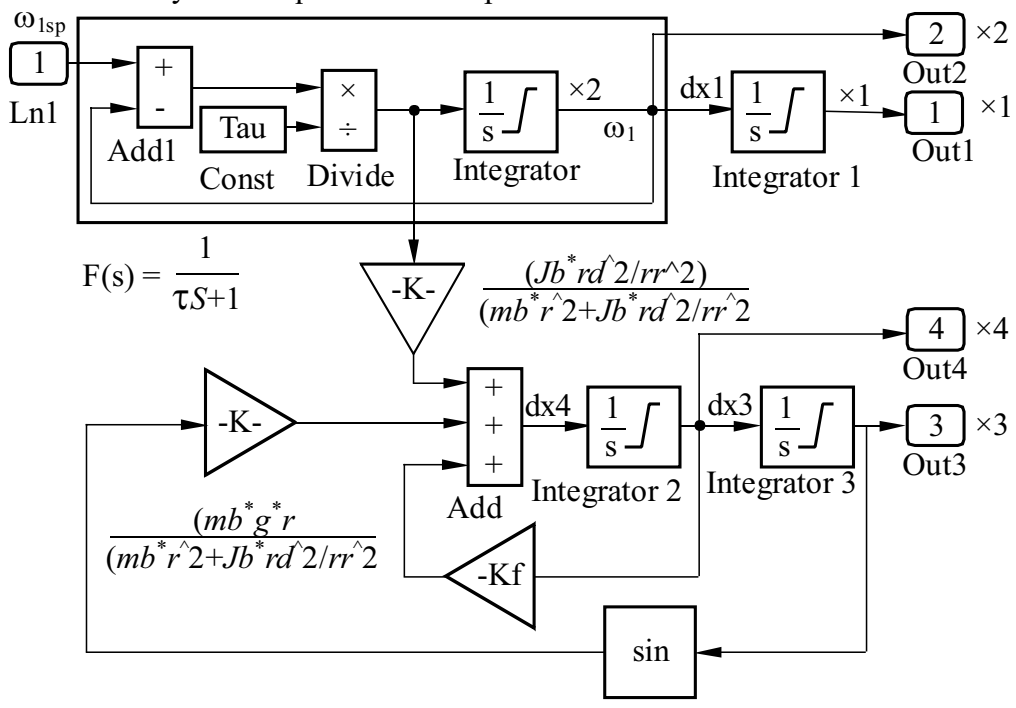

Fig. 3. Simulink scheme of simplified Ball on Spool model ('mic_simplified.mdl')

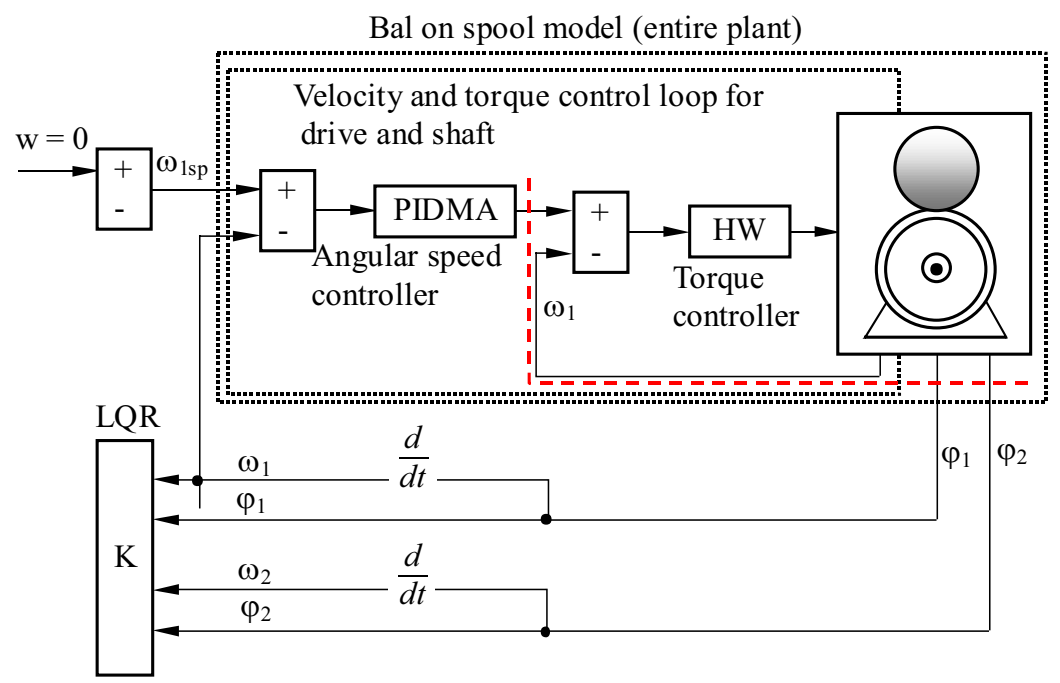

Fig. 4. Control loop with LQR controller

The numerical values of this matrix can also be verified by the use of Matlab command-line linmod function applied to Simulink scheme according Fig. 3, see the Matlab code listed below.

\section{DESIGN OF LQR CONTROLLER}

State-space description of simplified Ball on Spool model derived in previous section is a base for LQR design according Fig. 4. It is particularly based on lqr Matlab function, see [2] and [3].

Listing of Matlab code for LQR Controller Design $m b=0.405 ; r=0.15 \% ; r d=0.09 ; r r=r-r d ; r b=0.085 ; r b i=0.084$; $K f=0.3 ; g=9.81 ;$ tau $=0.015$;

$J b=2 / 5^{*} m b^{*}\left(r b^{\wedge} 5-r b i^{\wedge} 5\right) /\left(r b^{\wedge} 3-r b i^{\wedge} 3\right)$;

\%STATE-SPACE MATRIX A

$A=z \operatorname{eros}(4,4) ; B=z \operatorname{eros}(4,1)$;

$A(1,1)=0 ; A(1,2)=1 ; A(1,3)=0 ; A(1,4)=0 ; A(2,1)=$
$0 ; A(2,2)=0 ; A(2,3)=0 ; A(2,4)=0$;

$A(3,1)=0 ; A(3,2)=0 ; A(3,3)=0 ; A(3,4)=1 ; A(4,1)=0$; $A(4,2)=0$;

$A(4,3)=m b^{*} g^{*} r /\left(m b^{*} r^{\wedge} 2+J b^{*} r d^{\wedge} 2 / r r^{\wedge} 2\right) ; A(4,4)=-K f$; \%STATE-SPACE MATRIX B

$B(1,1)=0 ; B(2,1)=1 ; B(3,1)=0 ; B(4,1)=$

$\%$ LQR design

$\left(J b^{*} r d^{\wedge} \mathscr{2} / r r^{\wedge} \mathscr{Q}\right) /\left(m b^{*} r^{\wedge} \mathscr{Z}+J b^{*} r d^{\wedge} \mathscr{2} / r r^{\wedge} \mathscr{Q}\right)$;

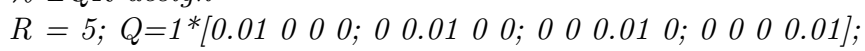
ss_entire=linmod('mic_simplified', [lllllll);

$[K, S, E]=l q r\left(s s \_\right.$entire.a,ss_entire. $\left.b, Q, R\right)$;

Above mentioned code results in determination of LQR controller: $K=\left[\begin{array}{llll}0.0447 & 2.0151 & -59.6861 & -8.5772\end{array}\right]$

\section{IMPLEMENTATION OF LQR CONTROLLER}

REX Control System is an advanced tool for design and implementation of complex control systems. Basically it consists of two parts: the development tools and the runtime sys- 

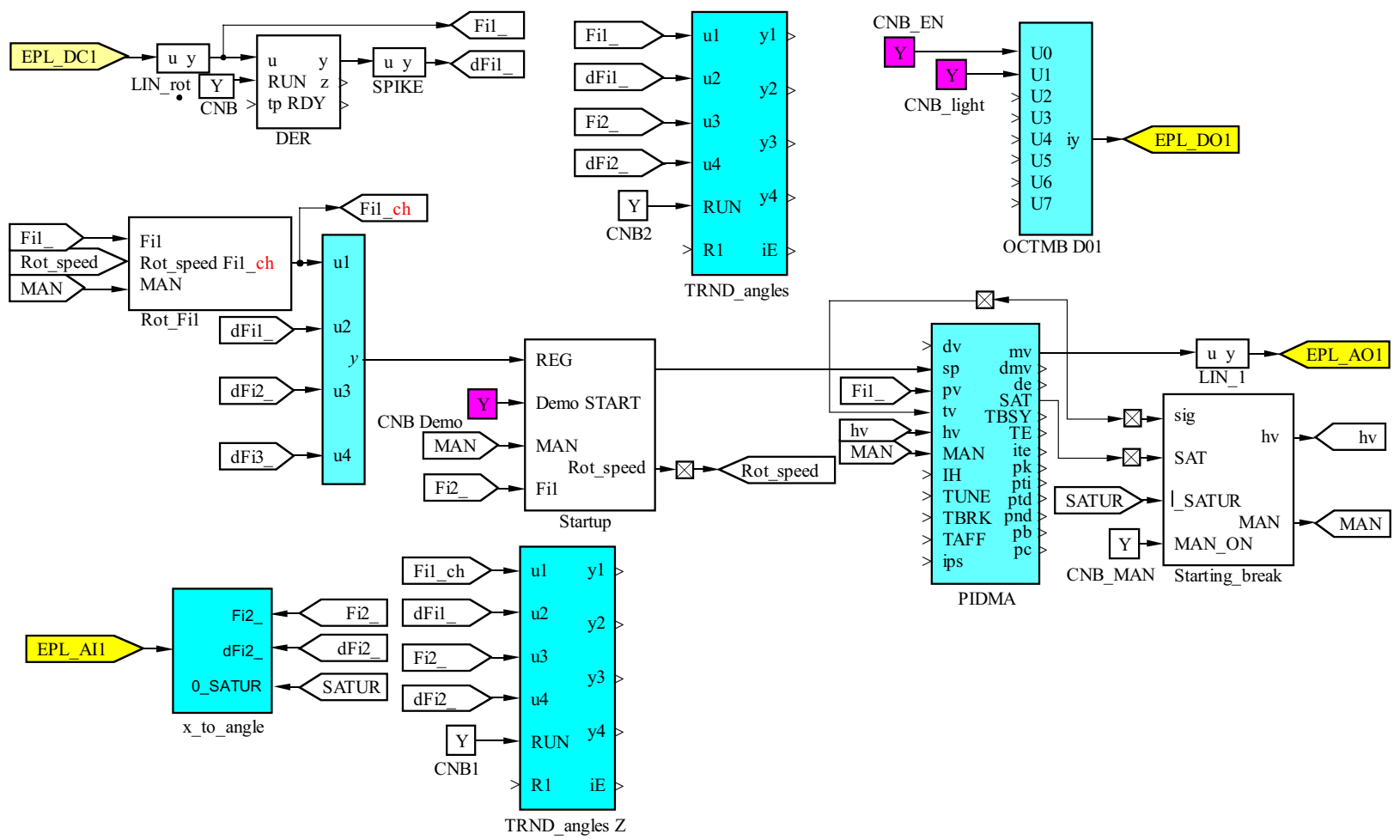

Fig. 5. Control scheme in RexDraw

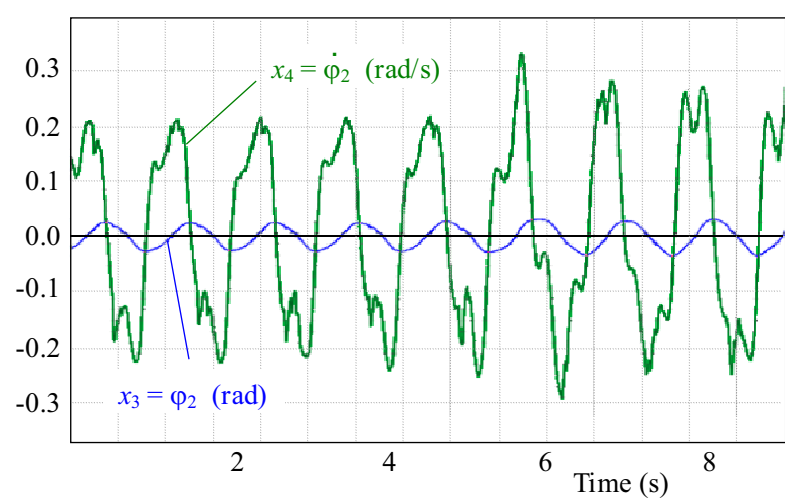

Fig. 6. Trends in RexView diagnostic utility

tem [4]. It is also compatible with Simulink environment, and the way of implementation of control algorithm is very similar, see Fig. 5. The control scheme is finally compiled (using RexDraw utility) and uploaded into the real-time target (using RexView utility). Structure of control loop according Fig. 4 contains one "internal" embedded hardware torque controller, one "external" automatically tuned PID controller represented by PIDMA block, and one state-space controller K. Moreover, the scheme contains all other necessary blocks for data processing and regulation. Interface between hardware and software parts is marked by a red dashed-line. Yellow "flags" in the scheme represents blocks for I/O interfaces, blue blocks stand for controllers. Principal situation of connecting real model with real-time target (single-board ALIX
2D13 with Linux/Xenomai) and I/O modules B-R X20 was shown in Fig. 1.

So far, at the Laboratory of cybernetics, there have been more educational models, most of them running on REX Control System since 2009. Particularly, it is Magnetic Levitation, Ball on Beam, Helicopter Model, Rotary Inverted Pendulum, Linear Inverted Pendulum, Three Tanks Model and others. Experience with similar LQR design for Inverted Pendulum model is documented in [3].

\section{DIAGNOSTICS, VERIFICATION AND MEASUREMENTS}

RexView utility is the main tool for online diagnostic and access to the running target. Besides a lot of other functions, it allows reading/writing access to all variables, including a basic visualization in the form of trends, see Fig. 6 that represents trends of $\varphi_{1}$ and $\varphi_{2}$ during regulation of ball position on the top of the spool. It is possible to change the parameters of all blocks in the scheme, especially the gains of statespace controller, and to see how they affect the dynamics of this educational model. Measured data can be easily exported for further analysis. Concept of REX Control system enables more TCP/IP connections of more users at the same time, either over RexView utility, SCADA/HMI programs or HTML5 visualization using a web browser. 


\section{VISUALIZATION BASED ON HTML5 STANDARD}

REX Control system allows automatically generated HTML5 visualization running on the same target as control algorithm, using lighttpd server. It enables user to read the online values at inputs/outputs of particular blocks and to set and change block parameters. It can also be combined with other graphical components whose parameters are directly connected to control variables, or with online generated trends. The main screen of automatically generated web visualization has the same look as the original scheme in RexDraw, and it can be customized and extended by adding any other text or graphic elements. Next installation of REX Control system is possible in Smart Home system, as is referred in [5] and [6].

Practical video example of functionality of the educational model of Ball and Spool can be seen through web link stated in [7]. It shows regulation of the ball in upright position, while later on there comes an external disturbance signal induced by user, trying to divert the ball off the set-point position.

\section{CONCLUSIONS}

The paper presents quite a unique, complex and modern educational model of nonlinear mechatronic system, used for lectures of automation courses at the Department of Cybernetics and Biomedical Engineering by VSB-Technical university of Ostrava. It presents connection between theoretical background and practical implementation by advanced REX Control system.

Currently, the educational model of Ball on Spool is ready for further research in the field of application of other types of modern control algorithms, such as robust control, adaptive control, predictive control or fuzzy control, as the REX Control System provides ultimate libraries for basic and advanced regulation, signal processing, logic control or motion control. REX Control System has been designed as a professional real-time control system for industrial applications, and successfully applied in tens of complex plants in practice, with excellent ratio performance/costs and possibility to simulate behavior of control loop before deployment on a real system.

Compatibility with Simulink, ease of use, reliable performance, supported multi-platforms, wide communication and data acquisition possibilities make it an ideal tool for educational process, too. It helps to develop laboratory background used for modern education, for example so called virtual laboratories with a remote access through the web, supporting mobile phones and tablets. Last but not least, it also supports low-cost solutions, for example Raspberry connected to Arduino boards, and is thus affordable to all students and public.

Close future works will be focused on application of other types of controllers based on so called modern control theory, and on more precise determination of time constant? which turned out to be one of the most crucial parameters influencing dynamic behavior of closed control loop.

\section{Acknowledgment}

This work was supported by project SP2015/154, "Development of algorithms and systems for control, measurement and safety applications" of Student Grant System, VSB-TU Ostrava

\section{REFERENCES}

[1] REX Controls s.r.o. Model míče na rotující cívce RBM-210. user guide, revision 1. Plzeň, September 2013.

[2] TEWARI, A.: Modern Control Design with MATLAB and SIMULINK, Wiley, Chichester, 2002.

[3] OZANA, S.-PIES, M.-SLANINA, Z.-HAJOVSKY, R. : Design and Implementation of LQR Controller for Inverted Pendulum by Use of REX Control System, $12^{\text {th }}$ International Conference on Control, Automation and Systems, ICCAS 2012, Jeju (2012), pp. 343-347.

[4] Information on http://www.rexcontrols.com/ .

[5] NOVAK, T.-VANUS, J.-SUMPICH, J.-KOZIOREK, J.SOKANSKY, K.-HRBAC, R. : Possibility to Achieve the Energy Savings by the Light Control in Smart Home, Proceedings of the $7^{\text {th }}$ International Scientific Symposium on Electrical Power Engineering (Elektroenergetika 2013), pp. 260-263, https://www.youtube.com/watch? $\mathrm{v}=\mathrm{f} 2 \mathrm{TezPul} 1 \mathrm{eQ}$.

[6] VANUS, J.-KOZIOREK, J.-HERCIK, R.: The Design of the Voice Communication in Smart Home Care, $36^{\text {th }}$ International Conference on Telecommunications and Signal Processing (TSP), 2013, pp. 561-564.

[7] https://www.youtube.com/watch?v=f2TezPul1eQ.

Received 11 December 2014

Štěpán Ožana, Martin Pieš, Radovan Hájovský and Tomáš Dočekal, biographies not supplied.

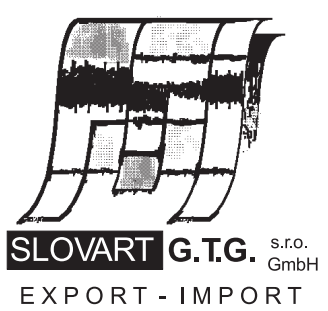

EXPORT - IMPORT
E X P OR T - I M P OR T

of periodicals and of non-periodically printed matters, books and CD-ROMs

Krupinská 4 PO BOX 152, 85299 Bratislava 5, Slovakia tel: ++421 263839 472-3, fax: ++421263839485 info@slovart-gtg.sk; http://www.slovart-gtg.sk

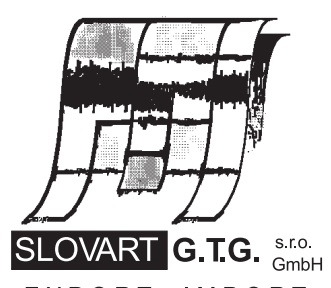

EXPORT - IMPORT 